\section{The molecule in print}

\section{Gary Ashley}

ChemText 1.2. Molecular Design, 2132 Farallon Drive, San Leandro, California 94577/Armstrong Mall, Unit B10, Southwood Summit Centre, Farnborough, Hampshire GU14 0NR. \$1,500, £1,500 (industry); $\$ 500, £ 750$ (academic).

ChemText is an integrated chemical structure-drawing and word-processing package that allows the user to produce chemical documents on an IBM-PC computer or compatible. Unlike most other chemical software packages, text and graphics are controlled within one program and can be displayed simultaneously on the screen, allowing great ease and flexibility in document design.

While its word-processing capabilities compare favourably with 'standard' programs, ChemText is unusual among IBM-PC word processors in that it has been designed with the non-technical user in mind. It is almost exclusively mousedriven with pull-down menus, so that there are no complex control codes to memorize or search for on a keyboard template. The program has a hierarchical

ADVERTISEMENT

\section{Journanals}

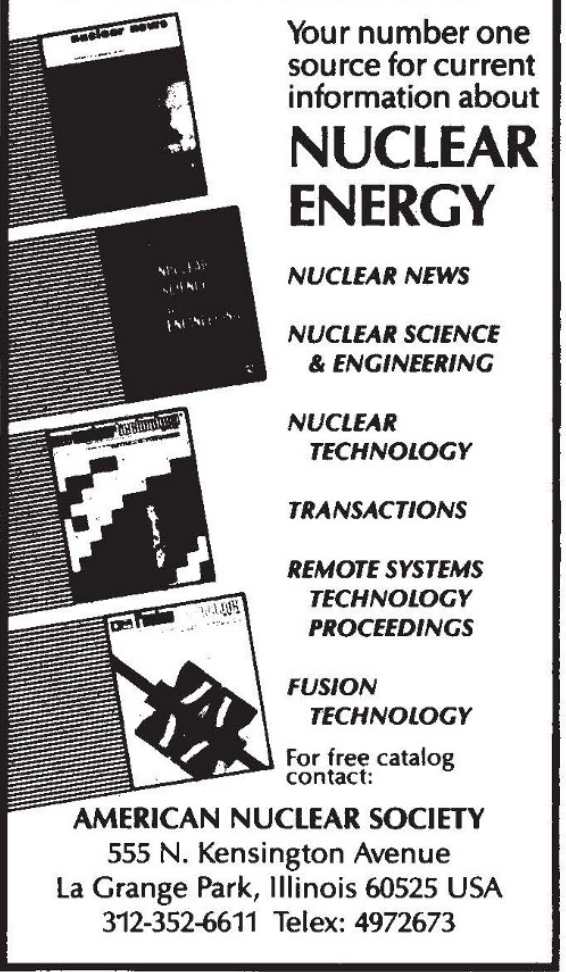

Reader Service No.31

structure, separating the three basic tasks of molecule drawing, sketch editing and word processing into separate modules.

Individual molecules are drawn and manipulated at the innermost level of the program. ChemText provides several commonly used partial structures, or templates, to aid in this process, and allows for storage of user-defined templates. Stereochemical bonds are readily

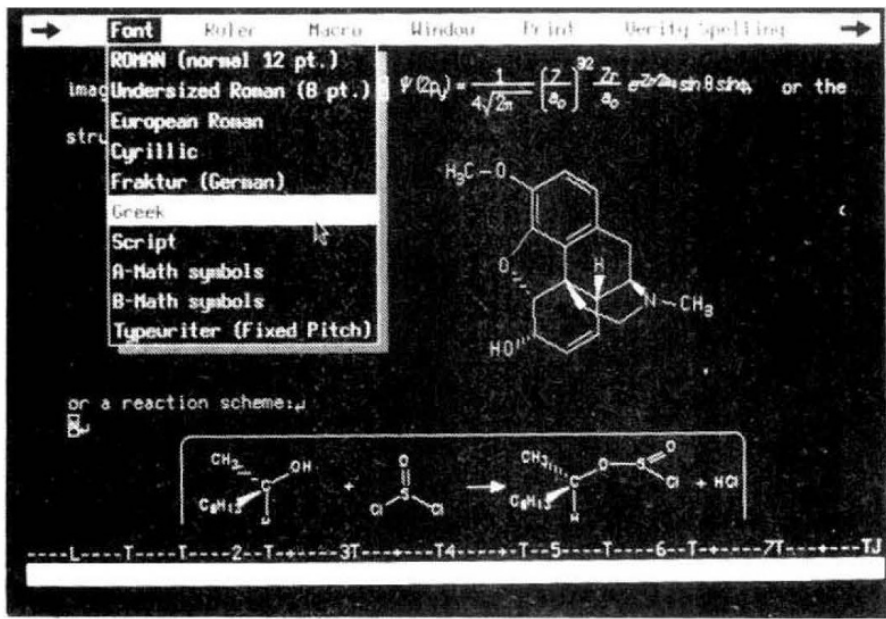

On the menu - Chem Text's choice of fonts pulled down onto work in progress on the screen. indicated, and bonds may be highlighted for perspective views. The program automatically checks valence, and the user can choose to display no hydrogen atoms, only those hydrogens attached to heteroatoms, or those attached to terminal carbons as well, with the program calculating the correct number of hydrogens at each position. There are also helpful features to indicate charge and isotopic composition, and a 'clean-up' facility which evens out structures drawn freehand.

At the next level up, molecules are assembled into 'sketches', complete with arrows, arcs, explanatory text, boxes and other notations. Molecules may only be moved and re-sized at this level. Alignment in complex diagrams is aided by movable, ruled crosshairs, a feature often lacking in structure-editing programs. A notable advantage of this division of labour is that once a molecule is drawn in the lowest level, it is treated as an object to be manipulated in the sketch editor. The user can draw a molecule without regard to size or placement in a diagram, and then fit various structures together at will to form a complex diagram.

The highest level of the program is the word processor, where the sketches can be combined with text to produce a completed document. ChemText allows great flexibility in page layout, enabling drawings to be placed in line with the text or as block diagrams. Page spacing is calculated automatically, and diagrams too large to fit the remaining space on a partially filled page are moved to the next page; the user may select either to have the program fill in the resulting space with text or to leave it blank.

The word-processing part of the package has most of the features found in standard programs, such as macro capability, windowing, block commands, headers and footers, text string location, and spelling checking using either American, British, German or French dictionaries. Because the entire screen is displayed in graphics, ChemText shows subscripts, superscripts, boldface, italics and special font characters as they will be printed, without having to replace the video character PROM chip in the computer. A variety of fonts are available, including Roman, undersized Roman, European Roman ( $\AA$, ä, for example), Cyrillic, Fraktur, Greek, script and various mathematical symbols.

The true test of any software package of this type is its output quality. ChemText is compatible with a wide range of printers, including those of the dot-matrix and laser type. For superb 300 dots per inch output, a PostScript printer such as the Apple LaserWriter is needed, although quite good 150 dots per inch is obtained with non-PostScript printers. ChemText supports 300 dots per inch of full-page graphics on a Hewlett-Packard LaserJet Series II having $2 \mathrm{Mb}$ of memory; otherwise, only 32 square inches of 300 dots per inch graphics per page can be supported.

Although the program will theoretically run on any PC family member, various practicalities limit its effective use to the PC-AT. The power of full-screen graphics has its price - the program takes up nearly $2 \mathrm{Mb}$ of disk space and cannot be run from floppy disks. The extra speed of the 80286 is really needed. Even on a $10 \mathrm{MHz}$, zerowait AT system, a pause long enough to be annoying accompanies image-drawing during scrolling in the text editor, and one might well die of old age during this process on a PC-XT.

ChemText is a top-of-the-line word processor for chemists. It is easy to learn and use, making it well worth its considerable expense. The program combines the best elements of the Macintosh user interface with the attributes of the IBM-PC AT. In terms of power and ease of use, there are really no software packages available which can compare with this one.

Gary Ashley is in the Department of Chemistry Northwestern University, Evanston, Illinois 60208, USA. 